\title{
RECONSTRUCTION OF SATELLITE IMAGES USING MODIFIED STATISTICAL ITERATIVE TECHNIQUE
}

\author{
Ayad A. A1-Ani \\ Department of Physics, College of Science, Al-Nahrain University, Jadriyah, Baghdad, Iraq. \\ E-mail: drayadalani61@yahoo.com, ayadalani1961@yahoo.c
}

\begin{abstract}
\section{Introduction}

In its most general image restoration or reconstruction refers to the correction of visible defects in observed images. Here, we consider an important subclass of image reconstruction problems and attempt to reverse known systematic distortions that can quantified in terms of spatial domain impulse or the blurring function " $\mathbf{d}(\mathbf{x}, \mathbf{y})$ ", or a frequency domain transfer function "D(u,v)". Several simple methods exist for removing these known distortions from a noiseless image, including direct and pseudo-inverse calculations. Unfortunately, under even moderate noise conditions, these methods may produce unacceptable results.
\end{abstract}

Modified Statistical Iterative Technique has been adapted to reconstruct satellite images. The adopted technique removes some of the assumptions used in the derivation of the Wiener filtering solution. Most obviously, the iterative approach we develop will require no assumptions about the underlying image generation process. The adapted filter was designed for reconstructing satellite images that are blurred with space-invariant blurring function corrupted with additive noise. Different degradation parameters, i.e. different width of blurring function and different signal to noise ratio were considered. The results using an adaptive technique were compared, quantitatively, using mean square error (MSE). Results shows that this method has better performance for reconstructing the degraded satellite images.

First, we formalize mathematically the degradation model used for this research. Consider an ideal (original) image denoted by $\mathrm{f}(\mathrm{x}, \mathrm{y})$ and a known distortion " $\mathbf{d}(\mathbf{x}, \mathbf{y})$ ". We model the distortion action using $[\mathbf{1 , 2 ]}$ : $\mathbf{g}(\mathbf{x}, \mathbf{y})=\mathbf{f}(\mathbf{x}, \mathbf{y}) \otimes \mathbf{d}(\mathbf{x}, \mathbf{y})+\mathbf{n}(\mathbf{x}, \mathbf{y})$

Where: $\mathbf{g}(\mathbf{x}, \mathbf{y})$ is the observed (recorded, degraded) image, and $\mathrm{n}(\mathrm{x}, \mathrm{y})$ is the noise signal. $\otimes$ represent circular convolution. Note that we have implicitly assumed an additive and uncorrelated noise. For much of the reminder of this work, we find it convenient to work in the frequency domain and therefore take the Discrete-time Fourier Transform (DTFT) on eq.(1) to arrive at:

$$
\mathbf{G}(\mathbf{u}, \mathbf{v})=\mathbf{F}(\mathbf{u}, \mathbf{v}) \mathbf{D}(\mathbf{u}, \mathbf{v})+\mathbf{N}(\mathbf{u}, \mathbf{v}) \ldots \text { (2) }
$$

The reconstructed image " $\hat{\mathbf{F}}(\mathbf{u}, \mathbf{v})$ ", can

be determined using:

$$
\begin{aligned}
& \hat{\mathbf{F}}(\mathbf{u}, \mathbf{v})=\mathbf{G}(\mathbf{u}, \mathbf{v}) \mathbf{H}(\mathbf{u}, \mathbf{v}) \\
& \hat{\mathbf{F}}(\mathbf{u}, \mathbf{v})=[\mathbf{F}(\mathbf{u}, \mathbf{v}) \mathbf{D}(\mathbf{u}, \mathbf{v})+\mathbf{N}(\mathbf{u}, \mathbf{v})] \mathbf{H}(\mathbf{u}, \mathbf{v})
\end{aligned}
$$

Image reconstruction may be therefore be recast as the problem of finding $\mathbf{H}(\mathbf{u}, \mathbf{v})$ such that $\hat{\mathbf{F}}(\mathbf{u}, \mathbf{v}) \cong \mathbf{F}(\mathbf{u}, \mathbf{v})$.

In the following section, we present a statistical reconstruction technique base upon the mathematical model of Wiener filtering. In section 3, we develop an iterative approach to solving the same problem. In section 4, we present the numerical and pictorial results, and their discussion. Finally, conclusion, have been presented in section five.

\section{Wiener Filter reconstruction}

An obvious flaw in the direct inverse method for image reconstruction (where $\mathbf{H}(\mathbf{u}, \mathbf{v})=\frac{\mathbf{1}}{\mathbf{D}(\mathbf{u}, \mathbf{v})}$ was chosen as the restoration filter) is the assumption that the ideal image is distorted in a noiseless way. Given some knowledge of the noise present in the observed image, it is reasonable to assume that we can develop a more effective reconstruction filter. Specifically, let us consider a case where certain statistical properties of the noise are known, let us 
assume access to the autocorrelation function and by extension the power spectral density "PSD" of the noise. Furthermore, we assume the autocorrelation and PSD of the ideal image are also known (in at least an approximate way) and that the ideal image and the observed image are jointly wide sense stationary stochastic process. Under these circumstances we may derive a Wiener filtering solution for the distortion model described in the introduction.

Let us define restoration filter $\mathbf{h}_{\mathbf{w}}(\mathbf{x}, \mathbf{y})$, where $x \in\left\{1,2, \ldots \ldots N_{1}\right\}$ and $y \in\left\{1,2, \ldots \ldots N_{2}\right\}$. Using this filter, we obtain a reconstructed image [3]:

$$
\hat{\mathbf{f}}(\mathbf{x}, \mathbf{y})=\mathbf{g}(\mathbf{x}, \mathbf{y}) \otimes \mathbf{h}_{\mathbf{w}}(\mathbf{x}, \mathbf{y})
$$

We call $\mathbf{h}_{\mathbf{w}}(\mathbf{x}, \mathbf{y})$ the Wiener filtering solution if it is the optimal filter in the sense of minimizing the mean squared error " $\varepsilon$ " between the reconstructed image and the ideal image. Mathematically [3]:

$\mathbf{h}_{\mathbf{w}}(\mathbf{x}, \mathbf{y})=\arg _{\mathbf{h}}(\mathbf{x}, \mathbf{y}) \min (\varepsilon)$

where:

$\varepsilon \equiv E\left[\sum_{x, y}\left(e(x, y)^{2}\right)\right]=E\left[\sum_{x, y}(f(x, y)-\hat{f}(x, y))^{2}\right] \ldots(6)$

We know the minimum MSE filter must satisfy the orthogonality principle, which stipulates that the expected error between the ideal and reconstructed images be (statistically speaking) orthogonal to each of the observed points used in forming the reconstructed image. This leads to the following conditions

$$
\mathbf{E}\left[(\mathbf{f}(\mathbf{x}, \mathbf{y})-\hat{\mathbf{f}}(\mathbf{x}, \mathbf{y})) \mathbf{g}\left(\mathbf{x}^{\prime}, \mathbf{y}^{\prime}\right)\right]=\mathbf{0} \quad \forall(\mathbf{x}, \mathbf{y}) \text { and }\left(\mathbf{x}^{\prime}, \mathbf{y}^{\prime}\right)
$$

Substituting in the expression from eq. (4) for our reconstructed image, we arrive at the following:

$$
\begin{aligned}
& \mathbf{E}\left[(\mathbf{f}(\mathbf{x}, \mathbf{y})-\hat{\mathbf{f}}(\mathbf{x}, \mathbf{y})) \mathbf{g}\left(\mathbf{x}^{\prime}, \mathbf{y}^{\prime}\right)\right]=\mathbf{0} \\
& \mathbf{E}\left[\left(\mathbf{f}(\mathbf{x}, \mathbf{y})-\mathbf{g}(\mathbf{x}, \mathbf{y}) \otimes \mathbf{h}_{\mathbf{w}}(\mathbf{x}, \mathbf{y})\right) \mathbf{g}\left(\mathbf{x}^{\prime}, \mathbf{y}^{\prime}\right)\right]=\mathbf{0}
\end{aligned}
$$

Which implies, using the linearity of the expectation operator, that:

$$
\begin{aligned}
& E\left[\left(f(x, y) g\left(x^{\prime}, y^{\prime}\right)\right)\right]=E\left[g(x, y) \otimes g\left(x^{\prime}, y^{\prime}\right)\right] \otimes h_{w}(x, y) \\
& R_{f g}\left(x-x^{\prime}, y-y^{\prime}\right)=R_{g g}\left(x-x^{\prime}, y-y^{\prime}\right) \otimes h_{w}(x, y) . .10
\end{aligned}
$$
where $\mathbf{R}_{\mathbf{f g}}$ and $\mathbf{R}_{\mathbf{g g}}$ are correlation functions. We may pull $\mathbf{h}_{\mathbf{w}}(\mathbf{x}, \mathbf{y})$ out of the expectations as it is a deterministic signal.
Taking the DTFT of both sides of eq. (10) and solving yields [4]:

$$
\mathbf{H}_{\mathrm{w}}(\mathrm{u}, \mathrm{v})=\frac{\mathbf{P}_{\mathrm{fg}}(\mathrm{u}, \mathrm{v})}{\mathbf{P}_{\mathrm{gg}}(\mathbf{u}, \mathrm{v})}
$$

Where $\mathbf{P}_{\mathbf{f g}}$ and $\mathbf{P}_{\mathbf{g g}}$ are power spectra. Recalling from eq.(1) that the observed image $(\mathrm{g})$ is obtained by passing the ideal image (f) through a deterministic distortion filter (d) and adding uncorrelated noise (n), we may rewrite eq.(11) in a more useful form [3]:

$$
\mathbf{H}_{\mathbf{w}}(\mathbf{u}, \mathbf{v})=\frac{\mathrm{D}^{*}(\mathbf{u}, \mathrm{v})}{\mid \mathrm{D}(\mathrm{u}, \mathrm{v})^{2}+\frac{\mathbf{P}_{\mathbf{n}}(\mathbf{u}, \mathrm{v})}{\mathbf{P}_{\mathbf{f}}(\mathbf{u}, \mathrm{v})}}
$$

Here $\mathbf{P}_{\mathbf{n}}(\mathbf{u}, \mathbf{v})$ and $\mathbf{P}_{\mathbf{f}}(\mathbf{u}, \mathbf{v})$ are the PSD's of the ideal image and the uncorrelated noise, respectively. Eq.(12) is the general form of the Wiener filtering solution and has a natural and attractive interpretation. Wherever $\mathbf{P}_{\mathbf{f}}$ is large relative to $\mathbf{P}_{\mathbf{n}}$, the ideal image has a large spectral component that should be preserved and reconstructed. In this case:

$$
\mathbf{H}_{\mathbf{w}}(\mathrm{u}, \mathrm{v}) \cong \frac{\mathrm{D}^{*}(\mathrm{u}, \mathrm{v})}{|\mathrm{D}(\mathrm{u}, \mathrm{v})|^{\mathbf{2}}}=\frac{\mathbf{1}}{\mathrm{D}(\mathrm{u}, \mathrm{v})}
$$

And the Wiener filter is approximately equal to the direct inverse solution. When $\mathbf{P}_{\mathbf{f}}$ is small with respect to $\mathbf{P}_{\mathbf{n}}$, the Wiener filter response is near zero.

\section{Statistical Iterative Reconstruction}

In this section, we present a second reconstruction technique that removes some of the assumptions used in the derivation of the Wiener filtering solution in eq.(12). Most obviously, the iterative approach we develop will require no assumptions about the underlying image generation process. We will therefore not need to estimate the power spectrum of the ideal image nor will we require information regarding the noise present in the image. Let us being the development by defining a simple iteration [4]:

$\hat{f}_{k+1}(x, y)=\hat{f}_{k}(x, y)+\beta\left(\hat{f}_{k}(x, y)\right)$.

where: $\quad \beta\left(\hat{\mathbf{f}}_{\mathbf{k}}(\mathbf{x}, \mathbf{y})\right)=\mathbf{g}(\mathbf{x}, \mathbf{y})-\mathbf{d}(\mathbf{x}, \mathbf{y}) \otimes \hat{\mathbf{f}}_{\mathbf{k}}(\mathbf{x}, \mathbf{y})$, $\beta$ is a strictly positive constant, and $\hat{\mathbf{f}}_{\mathbf{k}+\mathbf{1}}(\mathbf{x}, \mathbf{y})$ is the reconstructed image after 
$\mathrm{k}$ iterations. We may make a weak argument for the possible effectiveness of iteration by noting that $\mathbf{f}(\mathbf{x}, \mathbf{y})$, our ideal reconstructed image is a fixed point of iteration. Unfortunately, we cannot state with confidence that $\mathbf{f}(\mathbf{x}, \mathbf{y})$ is the only fixed point in the noiseless case nor can we be sure that the fixed point or points are anywhere near $\mathbf{f}(\mathbf{x}, \mathbf{y})$ in the presence of noise.

To investigate the conditions under converges to some image $\mathbf{f}_{\infty}(\mathbf{x}, \mathbf{y})$, we first take the DTFT of both sides in eq. (13) yielding:

$$
\hat{\mathbf{F}}_{k+1}(\mathrm{u}, \mathrm{v})=\beta \mathrm{G}(\mathrm{x}, \mathrm{y})+[1-\beta \mathrm{D}(\mathrm{u}, \mathrm{v})] \hat{\mathrm{F}}_{\mathrm{k}}(\mathrm{u}, \mathrm{v})
$$

We assume our initial image to be $\hat{\mathbf{f}}_{\mathbf{0}}(\mathbf{x}, \mathbf{y})=\mathbf{0} \quad \forall \mathbf{x}, \mathbf{y}$ with associated frequency domain representation $\hat{\mathbf{F}}_{\mathbf{0}}(\mathbf{x}, \mathbf{y})=\mathbf{0} \quad \forall \mathbf{u}, \mathbf{v}$. From this starting point, we may calculate our reconstructed image at each iteration recursively [5]:

$$
\begin{aligned}
& \hat{\mathbf{F}}_{\mathbf{1}}(\mathbf{u}, \mathbf{v})=\beta \mathbf{G}(\mathbf{u}, \mathbf{v}) \\
& \hat{F}_{2}(u, v)=\beta G(u, v)+[1-\beta D(u, v)] \beta G(u, v) \\
& \hat{\mathbf{F}}_{3}(\mathbf{u}, \mathbf{v})=\boldsymbol{\beta} \mathbf{G}(\mathbf{u}, \mathbf{v})+[1-\boldsymbol{\beta D}(\mathbf{u}, \mathbf{v})] \boldsymbol{\beta} \mathbf{G}(\mathbf{u}, \mathbf{v}) \\
& +[1-\boldsymbol{\beta D}(\mathbf{u}, \mathbf{v})]^{2} \boldsymbol{\beta} \mathbf{G}(\mathbf{u}, \mathbf{v})
\end{aligned}
$$

i.e. the reconstructed image after $\mathrm{k}$ iterations is given by:

$$
\hat{\mathbf{F}}_{k}(\mathrm{u}, \mathrm{v})=\beta G(\mathrm{u}, \mathrm{v}) \sum_{\mathrm{m}=\mathbf{0}}^{\mathrm{k}-1}[1-\beta D(\mathrm{u}, \mathrm{v})]^{\mathrm{m}} \ldots
$$

From eq.(16), we can easily glean $\mathbf{H}_{\mathbf{k}}(\mathbf{u}, \mathbf{v})$, the frequency response of the reconstruction filter after $\mathrm{k}$ iterations,

$$
H_{k}(u, v)=\beta \sum_{m=0}^{k-1}[1-\beta D(u, v)]^{m}
$$

To make some statement on the convergence properties of eqs. (16) and (17), we recall a few fundamental results from the calculus of infinite series. The sum [6]:

$$
S_{k}=\sum_{\mathbf{m}=\mathbf{0}}^{k-1} \mathbf{a}^{k}
$$

Where: $\mathrm{a}$ is in general complex valued, surely converges as $\mathbf{k} \rightarrow \infty$ if $|\mathbf{a}|\langle\mathbf{1}$. When this condition is satisfied, we get [6]: $\lim _{\mathbf{k} \rightarrow \infty} \mathbf{S}_{\mathbf{k}}=\frac{1}{1-\mathbf{a}}$

Applying this sufficient condition to eq. (16), we see that iteration converges if: $|\mathbf{1}-\beta \mathrm{D}(\mathrm{u}, \mathrm{v})|<\mathbf{1}$ by [7]:

The final reconstructed image is given

$$
\begin{aligned}
& \hat{\mathrm{F}}_{\infty}(\mathrm{u}, \mathrm{v})=\beta \mathrm{G}(\mathrm{u}, \mathrm{v}) \frac{1}{1-[1-\beta D(\mathrm{u}, \mathrm{v})]}=\frac{\mathrm{G}(\mathrm{u}, \mathrm{v})}{\mathrm{D}(\mathrm{u}, \mathrm{v})} \\
& \mathrm{H}_{\infty}(\mathrm{u}, \mathrm{v})=\frac{1}{\mathrm{D}(\mathrm{u}, \mathrm{v})} \ldots \ldots \ldots \ldots \ldots \ldots(21
\end{aligned}
$$

Which is the same result as would be attained when using the direct inverse. Note that the convergence condition in eq. (20) is not satisfied if $\mathbf{D}(\mathbf{u}, \mathbf{v})$ has a spectral null. Since $\mathbf{H}_{\mathbf{k}}(\mathbf{u}, \mathbf{v})$ in some cases grow to infinity in the limit, we must always stop the reconstruction process after a finite number of iterations. So the final reconstruction filter will have a large, but finite response wherever $\mathbf{D}(\mathbf{u}, \mathbf{v})$ has a spectral null. Furthermore, we have the option of stopping the iterative process whenever we arrive at satisfactory reconstruction and before observation noise.

We may also slightly modify eq.(13) so that it will converge even when $\mathbf{D}(\mathbf{u}, \mathbf{v})$ has a spectral null. We accomplish this by redistorting the observed image $\mathbf{G}(\mathbf{u}, \mathbf{v})$ with the complex conjugate of the distortion function $\mathbf{D}^{*}(\mathbf{u}, \mathbf{v})$ and using this new image as the starting point for our iteration. Let us define the redistorted image " $\mathbf{G}^{\prime}(\mathbf{u}, \mathbf{v}) "$ as [7]:

$$
\begin{aligned}
& G^{\prime}(u, v)=G(u, v) D^{*}(u, v) \\
& G^{\prime}(u, v)=F(u, v)|D(u, v)|^{2}+N(u, v) D^{*}(u, v)
\end{aligned}
$$

Redistorted image is now simply the ideal image distorted by $|\mathbf{D}(\mathbf{u}, \mathbf{v})|^{2}$. Accordingly, we modify eq.(13) to reflect this new distortion and arrive at (in the frequency domain):

$$
\hat{\mathrm{F}}_{\mathrm{k}+1}(\mathrm{u}, \mathrm{v})=\beta \mathrm{G}^{\prime}(\mathrm{u}, \mathrm{v})+\left[1-\beta \mid \mathrm{D}(\mathrm{u}, \mathrm{v})^{2}\right] \hat{\mathrm{F}}_{\mathrm{k}}(\mathrm{u}, \mathrm{v})
$$

We can determine a closed-form expression for both the reconstructed image and the reconstruction filter $\mathrm{k}$ iterations [7]: 


$$
\begin{aligned}
& \hat{\mathbf{F}}_{\mathrm{k}}(\mathrm{u}, \mathrm{v})=\beta \mathrm{G}^{\prime}(\mathrm{u}, \mathrm{v}) \sum_{\mathrm{m}=0}^{\mathrm{k}-1}\left[1-\beta \mid \mathrm{D}(\mathrm{u}, \mathrm{v})^{2}\right]^{\mathrm{m}} \\
& \hat{\mathbf{F}}_{k}(\mathrm{u}, \mathrm{v})=\beta \mathrm{G}(\mathrm{u}, \mathrm{v}) \mathrm{D}^{*}(\mathrm{u}, \mathrm{v}) \sum_{\mathrm{m}=0}^{\mathrm{k}-1}\left[1-\beta|\mathrm{D}(\mathrm{u}, \mathrm{v})|^{2}\right]^{\mathrm{m}} \\
& H_{k}(u, v)=\beta D^{*}(u, v) \sum_{m=0}^{k-1}\left[1-\beta|D(u, v)|^{2}\right]^{m}
\end{aligned}
$$

Eqs.(25) and (26) now converge if $\mathbf{D}^{*}(\mathbf{u}, \mathbf{v})=\mathbf{0}$ or $\left.|\mathbf{1}-\boldsymbol{\beta}| \mathbf{D}(\mathbf{u}, \mathbf{v})\right|^{\mathbf{2}} \mid\langle\mathbf{1}$, or in other form:

$$
0<\beta<\frac{1}{\max _{u, v}\left\{|D(u, v)|^{2}\right\}}
$$

In this case, we see that in the limit, the reconstruction filter given in eq.(26), will converge to:

$$
H_{\infty}(u, v)=\left\{\begin{array}{lc}
\frac{1}{D(u, v)} & \text { if } D(u, v) \\
0 & \text { otherwise }
\end{array}\right\} \ldots(28)
$$

This is sometimes referred to as the pseudo-inverse solution. Now, if $\mathbf{D}(\mathbf{u}, \mathbf{v})$ has spectral zeros, the ideal image cannot be uniquely recovered even under noiseless conditions. Clearly, spectral information about $\mathbf{F}(\mathbf{u}, \mathbf{v})$ is permanently deleted when the image is distorted.

Eqs. (25) and (26) are the fundamental results of this section. They will be used to generate the results presented in section 4 . We must emphasize that, although we have discussed the convergence properties of these iterations in some detail, this algorithm is not designed to run to convergence. It is instead intended to be monitored by a user who may qualitatively determine the best tradeoff between distortion correction and noise amplification.

\section{Results and Discussion}

A satellite grey image of $128 * 128$ pixels size, Baghdad satellite-image, as shown in figure (1-a), was used to check the quality of the statistical iterative technique:

The degraded (blurred and noisy) satellite images are simulated as follows:
1. The blurred images were simulated by convolving the original satellite image with circular function of radius $\mathbf{R}$, two values of $\mathbf{R}=1$ and 2 have been taken.

2. Random noise of Gaussian distribution with zero means was added to the blurred image (obtained in step 1). Different Signal to Noise ratio " SNR ", "NR = $10 \mathrm{~dB}, 20 \mathrm{~dB}, 30 \mathrm{~dB}, 40 \mathrm{~dB}$, and $50 \mathrm{~dB}$, have been taken.

Figure (1-b, to 1-f) shows the original image after degraded with circular blurring function of radius $(\mathbf{R}=\mathbf{1})$ and additive Gaussian noise with SNR equal to: $10 \mathrm{~dB}$, $20 \mathrm{~dB}, 30 \mathrm{~dB}, 40 \mathrm{~dB}$, and $50 \mathrm{~dB}$, respectively. The figure shows, also, the Mean Square Error (MSE) of the degraded image with respect to the original image.

Figure (2) shows the restored images and the corresponding MSE for the degraded image that blurred with circular blur of $\mathrm{R}=1$ and $\mathbf{S N R}=\mathbf{1 0} \mathrm{dB}$, after 1 iteration, 10 iterations, and 30 iterations, respectively.

Figure (3) shows the restored images and the corresponding MSE for the degraded image that blurred with circular blur of $\mathbf{R}=\mathbf{1}$ and $\mathbf{S N R}=\mathbf{2 0} \mathrm{dB}$, after 1 iteration, 10 iterations, and 30 iterations, respectively

Figure (4) shows the restored images and the corresponding MSE for the degraded image that blurred with circular blur of $\mathbf{R}=\mathbf{1}$ and $\mathbf{S N R}=\mathbf{3 0} \mathrm{dB}$, after 1 iteration, 10 iterations, and 30 iterations, respectively.

Figure (5) shows the restored images and the corresponding MSE for the degraded image that blurred with circular blur of $\mathbf{R}=\mathbf{1}$ and

$\mathbf{S N R}=\mathbf{4 0} \mathrm{dB}$, after 1 iteration, 10 iterations, and 30 iterations, respectively

Figure (6) shows the restored images and the corresponding MSE for the degraded image that blurred with circular blur of $\mathbf{R}=\mathbf{1}$ and $\mathbf{S N R}=\mathbf{5 0} \mathrm{dB}$, after 1 iteration, 10 iterations, and 30 iterations, respectively

Figure (7) shows the MSE for the restored images versus no. of iterations for the degraded image blurred with circular blur of $\mathbf{R}=1$ and with $\mathrm{SNR}=10 \mathrm{~dB}, 20 \mathrm{~dB}, 30 \mathrm{~dB}$, $40 \mathrm{~dB}$, and $50 \mathrm{~dB}$, respectively. 
Figure (8-b to and 8-f) shows the original image after degraded with circular blurring function of radius $(\mathbf{R}=\mathbf{2})$ and additive Gaussian noise with signal to noise ratio (SNR) are $10 \mathrm{~dB}, 20 \mathrm{~dB}, 30 \mathrm{~dB}, 40 \mathrm{~dB}$, and $50 \mathrm{~dB}$, respectively. The figure shows, also, the Mean Square Error (MSE) of the degraded image with respect to the original image.

Figure (9) shows the restored images and the corresponding MSE for the degraded image that blurred with circular blur of $\mathbf{R}=\mathbf{2}$ and $\mathbf{S N R}=\mathbf{1 0} \mathrm{dB}$, after 1 iteration, 10 iterations, and 30 iterations, respectively.

Figure (10) shows the restored images and the corresponding MSE for the degraded image that blurred with circular blur of $\mathbf{R}=\mathbf{2}$ and $\mathbf{S N R}=\mathbf{2 0} \mathrm{dB}$, after 1 iteration, 10 iterations, and 30 iterations, respectively

Figure (11) shows the restored images and the corresponding MSE for the degraded image that blurred with circular blur of $\mathbf{R}=\mathbf{2}$ and $\mathbf{S N R}=\mathbf{3 0} \mathrm{dB}$, after 1 iteration, 10 iterations, and 30 iterations, respectively.

Figure (12) shows the restored images and the corresponding MSE for the degraded image that blurred with circular blur of $\mathbf{R}=\mathbf{2}$ and $\mathbf{S N R}=\mathbf{4 0} \mathrm{dB}$, after 1 iteration, 10 iterations, and 30 iterations, respectively

Figure (13) shows the restored images and the corresponding MSE for the degraded image that blurred with circular blur of $\mathbf{R}=\mathbf{2}$ and $\mathbf{S N R}=\mathbf{5 0} \mathrm{dB}$, after 1 iteration, 10 iterations, and 30 iterations, respectively

Figure (14) shows the MSE for the restored images versus no. of iterations for the degraded image blurred with circular blur of $\mathbf{R}=\mathbf{2}$ and with $\mathrm{SNR}=10 \mathrm{~dB}, 20 \mathrm{~dB}, 30 \mathrm{~dB}$, $40 \mathrm{~dB}$, and $50 \mathrm{~dB}$, respectively.

\section{Conclusion}

In this paper, we construct an adaptive iterative technique for restoring the degraded (blurred and noisy) satellite images.

From the above Figures, we can conclude that the adopted technique is an efficient method to reconstruct the degraded images. We also, conclude that the mean square error of the reconstructed images decreases with the increasing the number of iterations until the result convergent.
Moreover, the convergence, mostly, happens after 10 iterations, and also decreased with increasing SNR. Finally, we can conclude that the ratio of the MSE of the degraded image to the corresponding reconstructed image at iteration 30 will increased with increasing SNR, i.e. this technique has better performance for less degradation parameters, i.e. with high SNR.

\section{References}

1- R.C. Gonzalez and P. Wintz, "Digital Image Processing", $2^{\text {nd }}$ ed., Addison Wisely Publishing Company (1989)

2- H.C. Andrews and B.R. Hunt, "Digital Image Restoration", Prentice - Hill Jersey (1977).

3- W.K.Pratt, Digital image Processing, $3^{\text {rd }}$ Ed., New York; John-Wiley and sons (2001)

4- A.Papoulis and S.V.Pillai, Probability, random variables and stochastic process, $4^{\text {th }}$ Ed., Boston, McGraw-Hill, (2002)

5- D.G.Manolakis, Statistical and Adaptive signal processing: Spectral estimation, signal modeling, adaptive filtering and array processing, Boston, McGrawHill, (2000)

6- C.R.Vogel, Computational Methods for inverse problems, SIAM, Philadelphia (2002)

7- D.P.Lun, T.C.Chan, T.C.Hsung, D. D. Feng, and Y.H.Chan; Efficient blind restor-ation using discrete periodic random transform; IEEE Trans. Image Processing 13 (2004) 188-200 


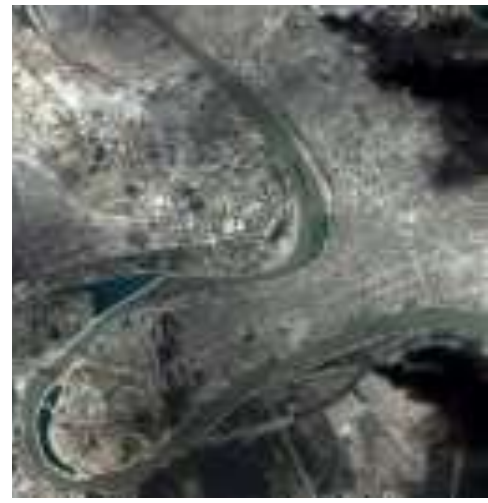

(a)

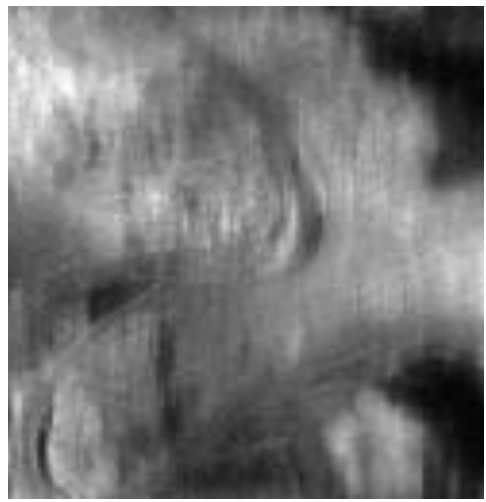

(d)

MSE $=\mathbf{5 0}$

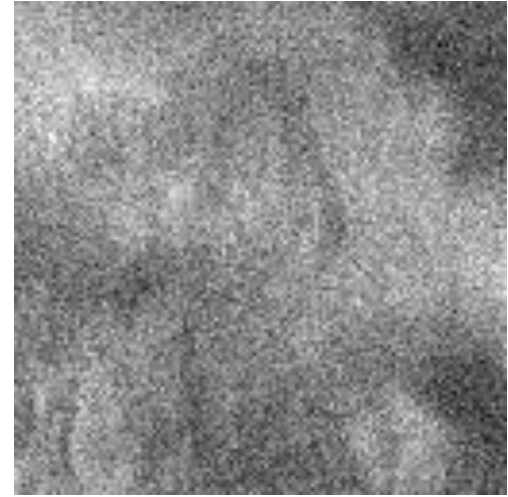

(b)

MSE $=\mathbf{4 3 7 . 7}$

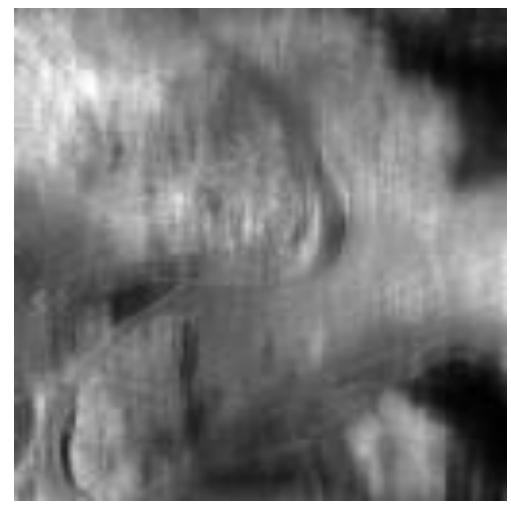

(e)

$\mathrm{MSE}=\mathbf{4 6 . 6}$

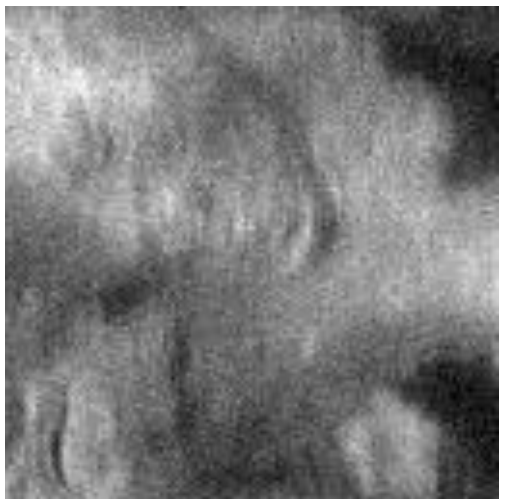

(c)

MSE $=87$

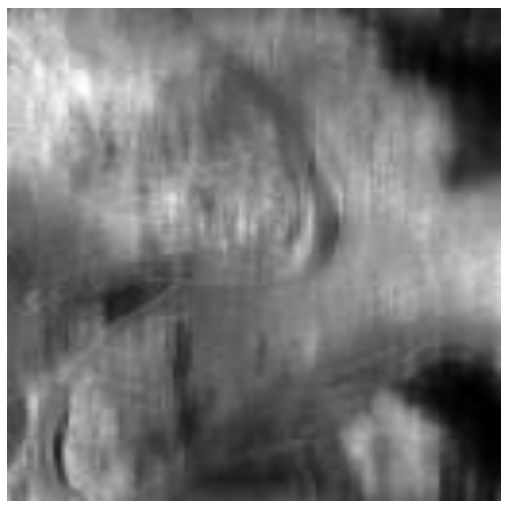

(f)

MSE $=46.2$

Fig.(1)

(a) Original image, "Baghdad" of size 128x128

(b) Degraded image with circular blur function of $R=1$ and with additive Gaussian noise with $\mathrm{SNR}=10 \mathrm{~dB}$

(c) Degraded image with circular blur function of $R=1$ and with additive Gaussian noise with SNR=20 dB

(d) Degraded image with circular blur function of $R=1$ and with additive Gaussian noise with $\mathrm{SNR}=30 \mathrm{~dB}$

(e) Degraded image with circular blur function of $R=1$ and with additive Gaussian noise with $\mathrm{SNR}=40 \mathrm{~dB}$

(f) Degraded image with circular blur function of $R=1$ and with additive Gaussian noise with $\mathrm{SNR}=50 \mathrm{~dB}$ 


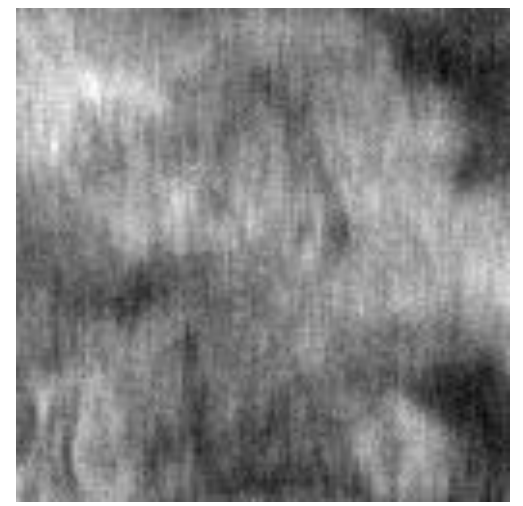

(a)

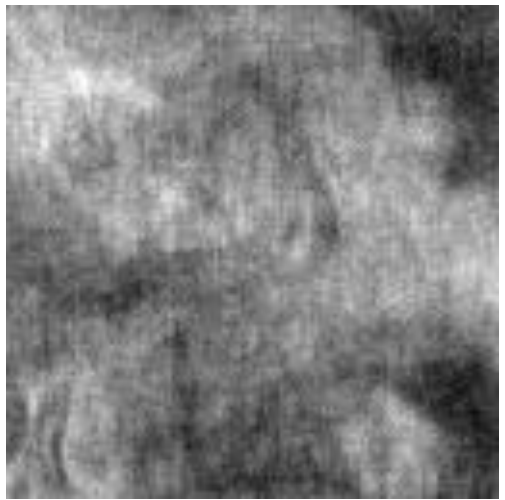

(b)

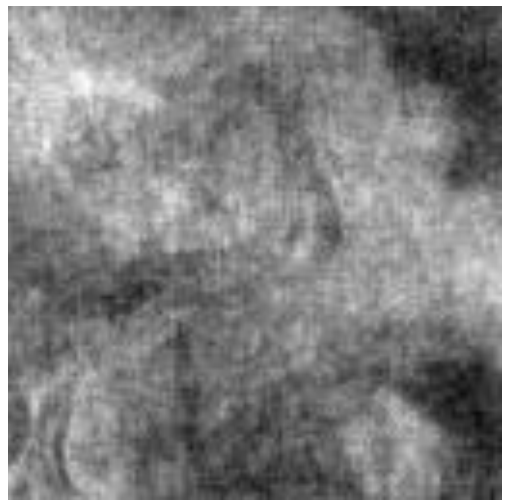

(c)

MSE $=114.6$

MSE $=104.5$

Fig.(2) Reconstructed images of Fig.(1-b) ( i.e. $R=1$ and $S N R=10 \mathrm{~dB}$ ) after:

$\begin{array}{lll}\text { (a) } 1 \text { iteration } & \text { (b) } 10 \text { iterations (c) } 30 \text { iterations }\end{array}$

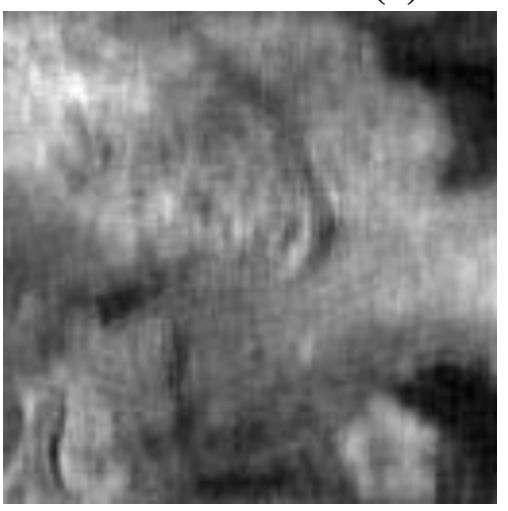

(a)

$\mathrm{MSE}=\mathbf{5 4 . 1}$

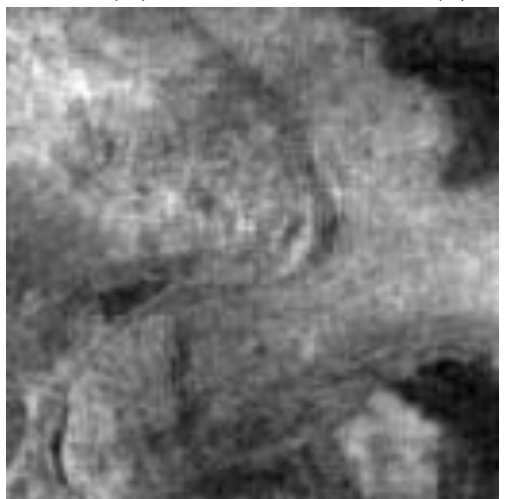

(b)

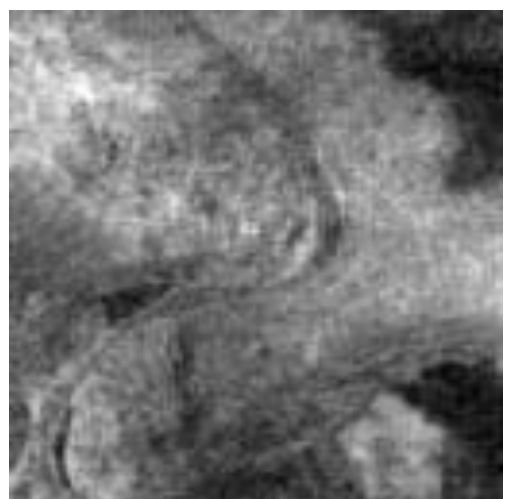

(c)

MSE $=45.7$

Fig.(3) Reconstructed images of Fig.(1-c) (i.e. $R=1$ and $S N R=20$ dB) after: $\begin{array}{lll}\text { (a) } 1 \text { iteration } & \text { (b) } 10 \text { iterations } & \text { (c) } 30 \text { iterations }\end{array}$

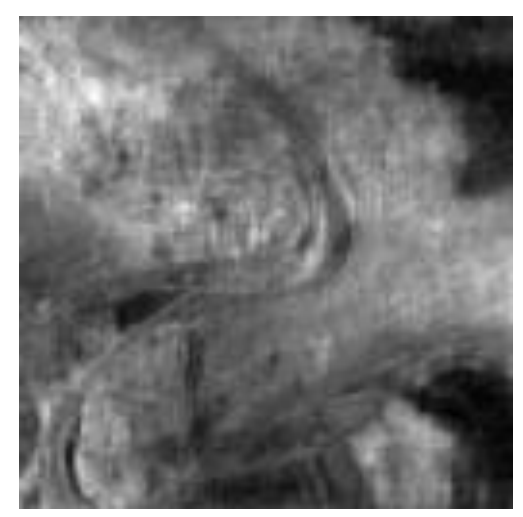

(a)

MSE $=\mathbf{2 7 . 5}$

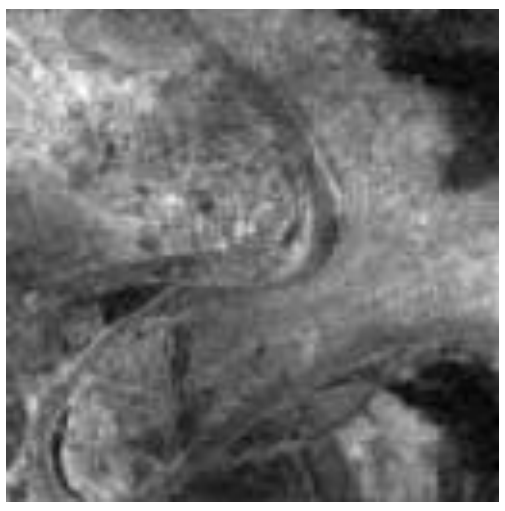

(b)

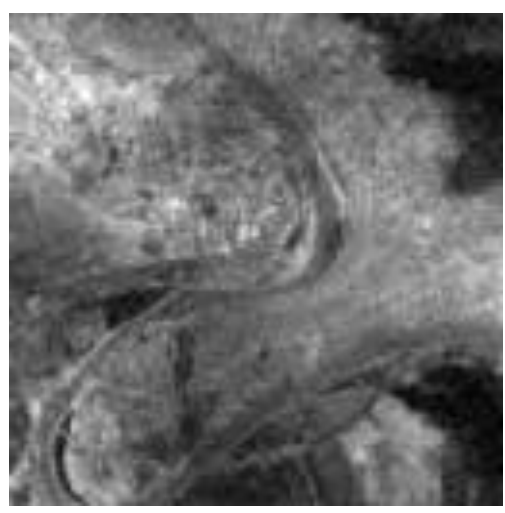

(c)

(b) $\mathrm{MSE}=17.1$

MSE $=16.6$

Fig.(4) Reconstructed images of Fig.(1-d) (i.e. $R=1$ and $S N R=30$ dB) after:
(a) 1-iteration
(b) 10 iterations
(c) 30 iterations 


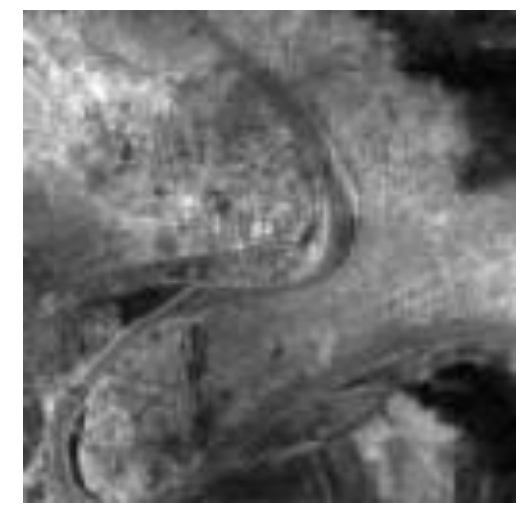

(a)

MSE $=9.4$

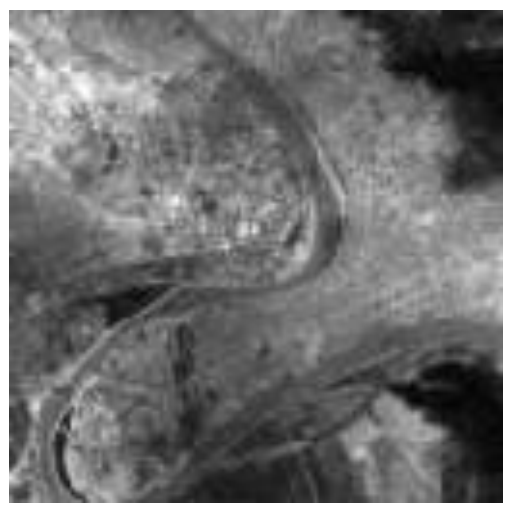

(b)

(b) $\mathrm{MSE}=3.9$

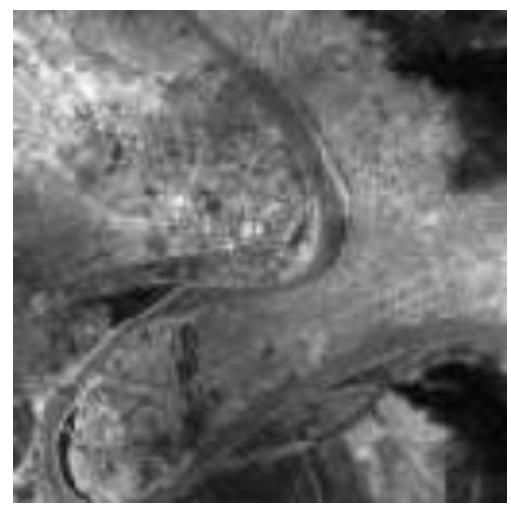

(c)

MSE $=3.7$

Fig.(5) Reconstructed images of Fig.(1-e) (i.e. $R=1$ and $S N R=40$ dB) after:

(a) 1 iteration

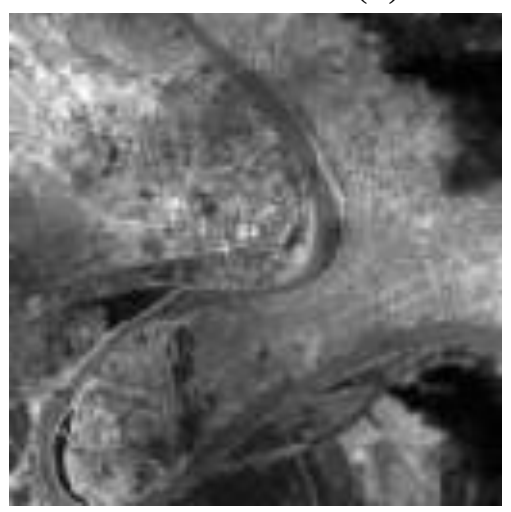

(a)

(b) 10 iterations

(c) 30 iterations

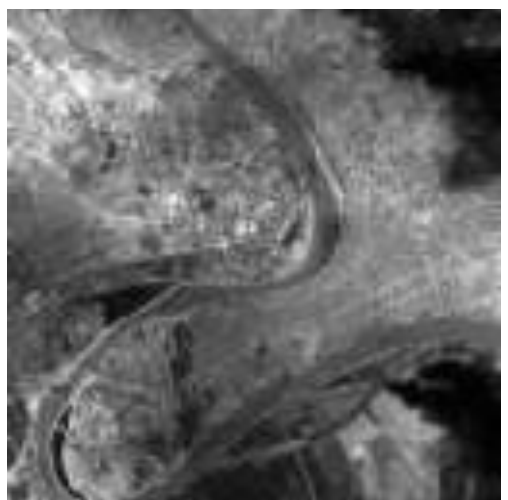

(b)

MSE $=1.5$

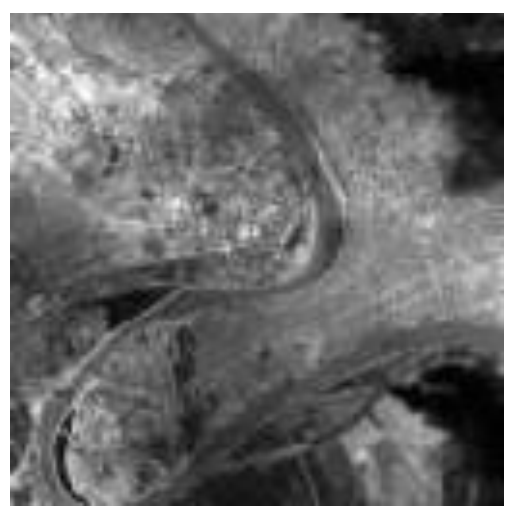

(c)

Fig.(6) Reconstructed images of Fig.(1-f) (i.e. $R=1$ and $S N R=50 \mathrm{~dB}$ ) after:
(a) 1 iteration
(b) 10 iterations
(c) 30 iterations 


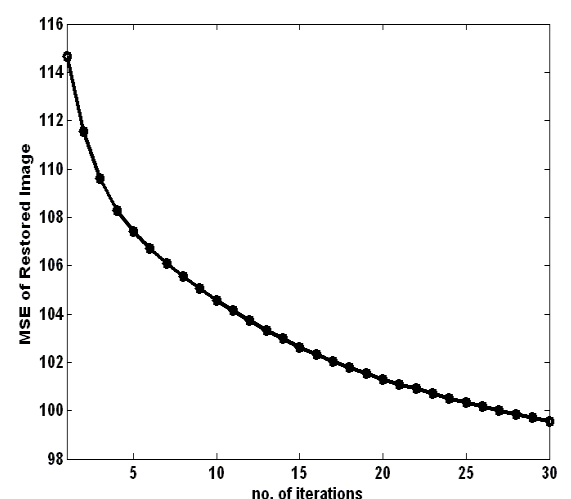

(a)

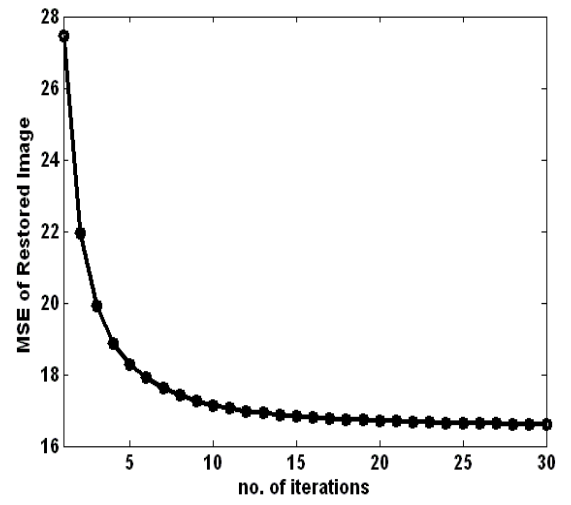

(c)

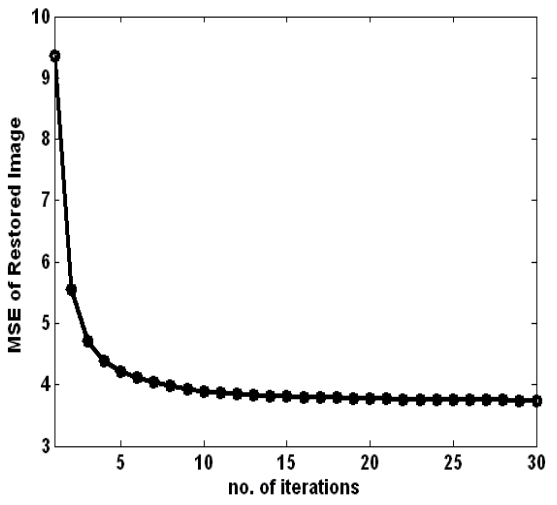

(d)

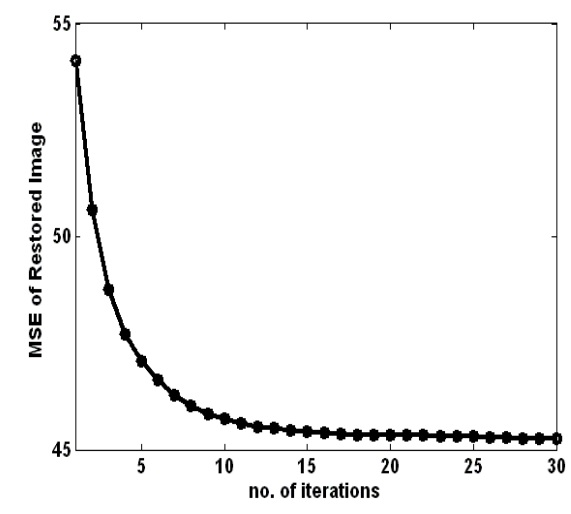

(b)

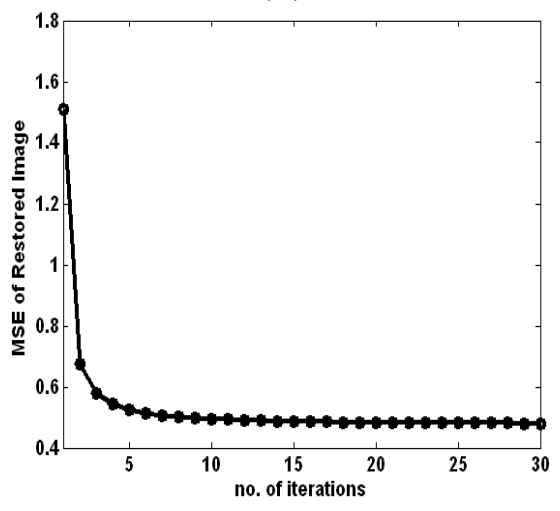

(e)

Fig. (7) Mean Square Errors (MSE) of the Reconstructed images Versus no. of iterations for:

(a) degraded Baghdad image, with circular function of $R=1$ and $S N R=10 \mathrm{~dB}$

(b) degraded Baghdad image, with circular function of $R=1$ and $S N R=20 \mathrm{~dB}$

(c) degraded Baghdad image, with circular function of $R=1$ and $S N R=30 \mathrm{~dB}$

(d) degraded Baghdad image, with circular function of $R=1$ and $S N R=40 \mathrm{~dB}$

(e) degraded Baghdad image, with circular function of $R=1$ and $S N R=50 \mathrm{~dB}$ 


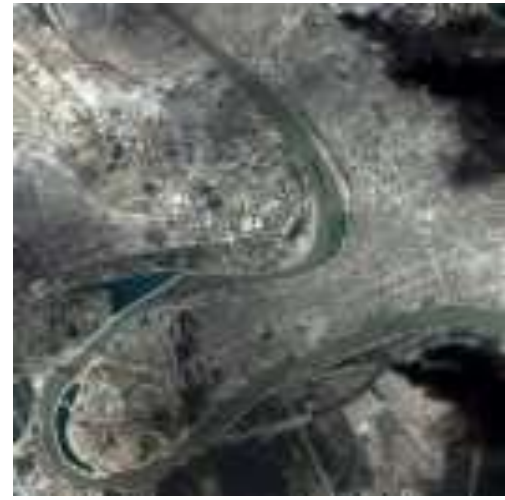

(a)

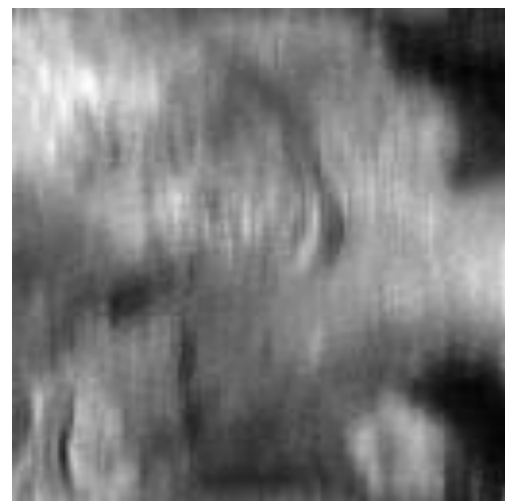

(d)

MSE $=74.3$

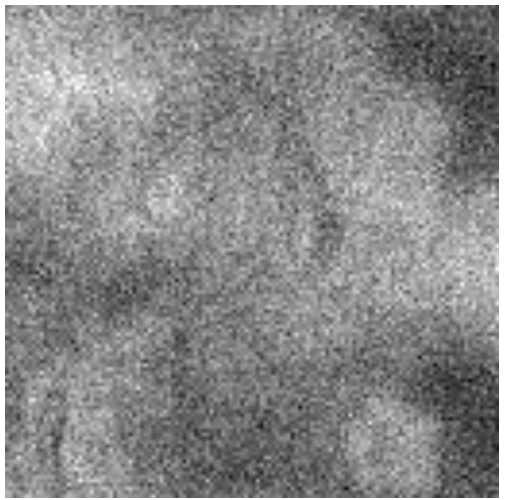

(b)

MSE $=\mathbf{4 7 4 . 2}$

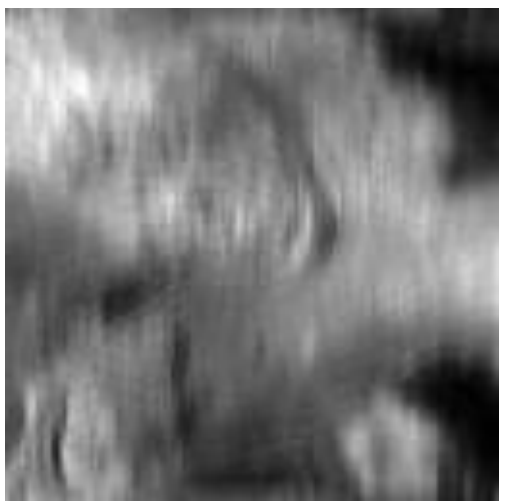

(e)

$\mathrm{MSE}=\mathbf{7 0 . 3}$

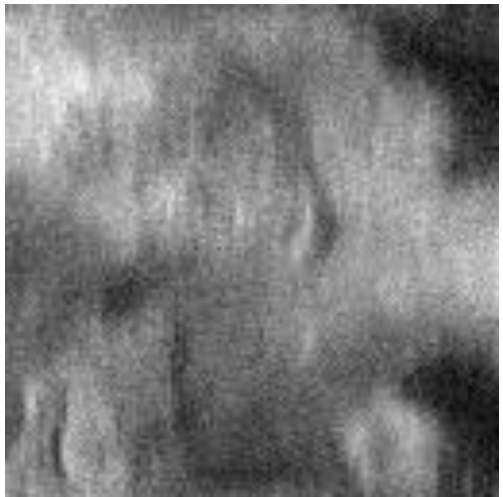

(c)

MSE $=109$

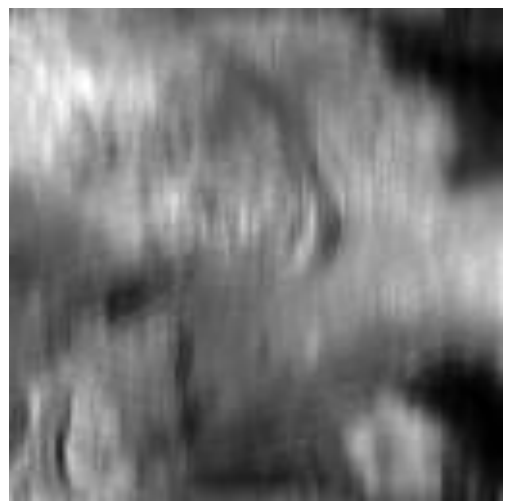

(f)

$\mathrm{MSE}=\mathbf{7 0}$

Fig.(8)

(a) Original image, " Baghdad " of size 128x128

(b) Degraded image with circular blur function of $R=2$ and with additive Gaussian noise with $\mathrm{SNR}=10 \mathrm{~dB}$

(c) Degraded image with circular blur function of $R=2$ and with additive Gaussian noise with $\mathrm{SNR}=20 \mathrm{~dB}$

(d) Degraded image with circular blur function of $\mathrm{R}=2$ and with additive Gaussian noise with SNR=30 dB

(e) Degraded image with circular blur function of $\mathrm{R}=2$ and with additive Gaussian noise with $\mathrm{SNR}=\mathbf{4 0} \mathrm{dB}$

(f) Degraded image with circular blur function of $R=2$ and with additive Gaussian noise with SNR $=50 \mathrm{~dB}$ 


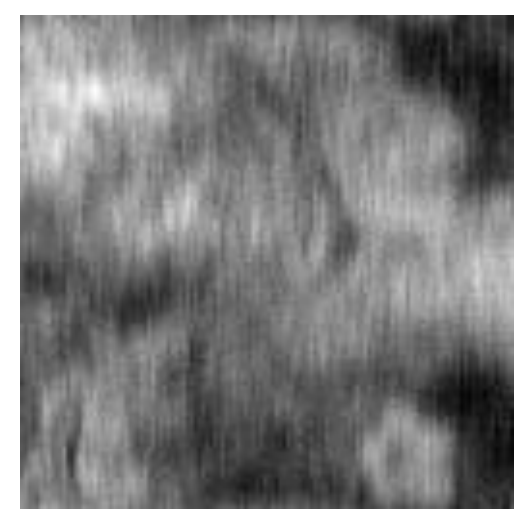

(a)

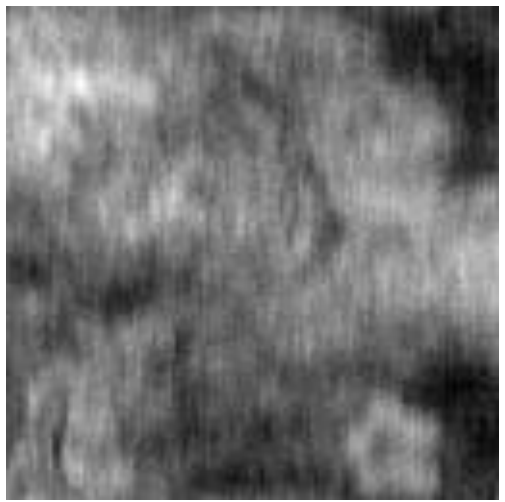

(b)

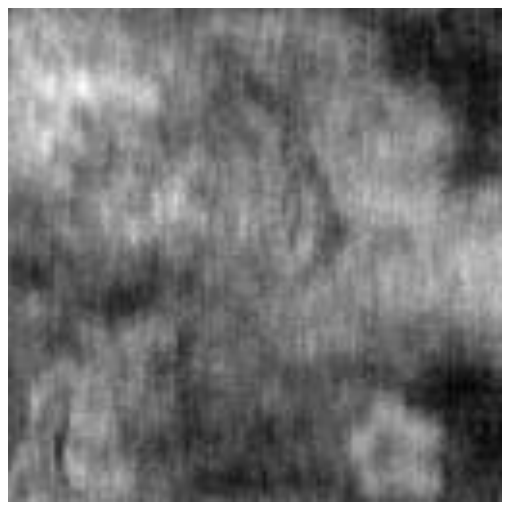

(c)

MSE $=115$

MSE $=108$

Fig.(9) Reconstructed images of Fig.(8-b) ( i.e. $R=2$ and $S N R=10 \mathrm{~dB}$ ) after:
(a) 1 iteration
(b) 10 iterations
(c) 30 iterations

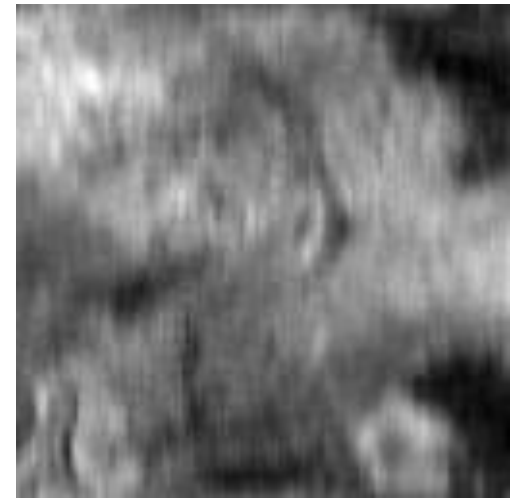

(a)

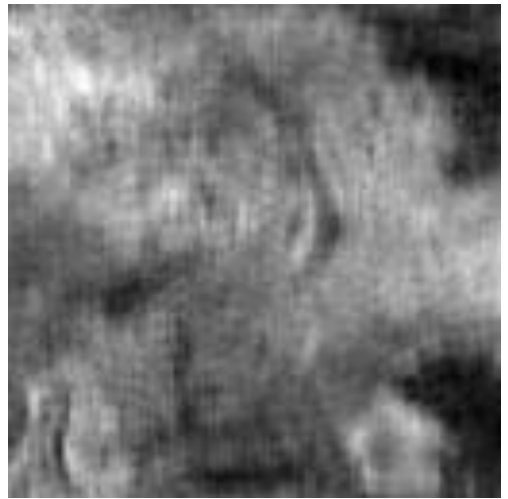

(b)

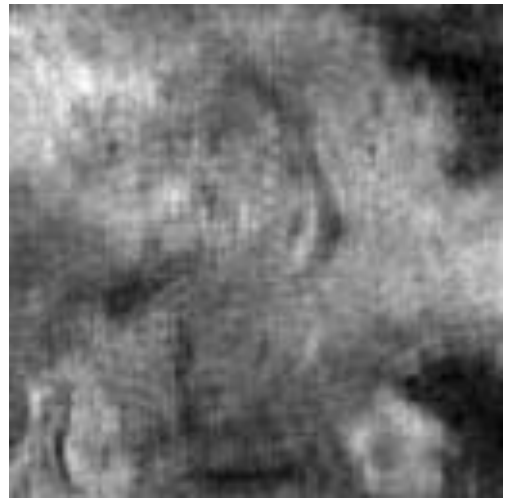

(c)

MSE $=73.5$

(b) MSE $=66.6$

MSE $=65.7$

Fig.(10) Reconstructed images of Fig.(8-c) ( i.e. $R=2$ and $S N R=20 \mathrm{~dB}$ ) after: $\begin{array}{lll}\text { (a) } 1 \text { iteration } & \text { (b) } 10 \text { iterations (c) } 30 \text { iterations }\end{array}$

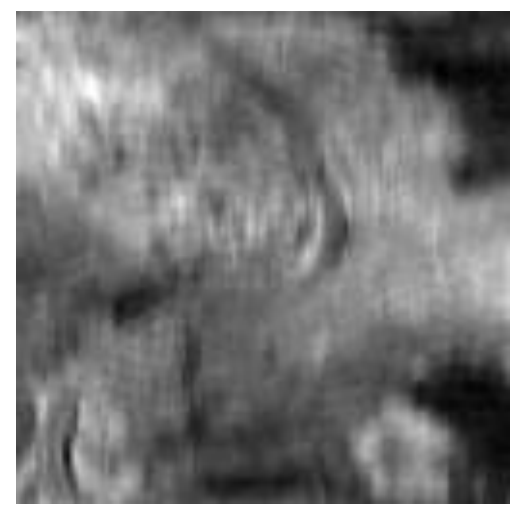

(a)

MSE $=\mathbf{5 7}$

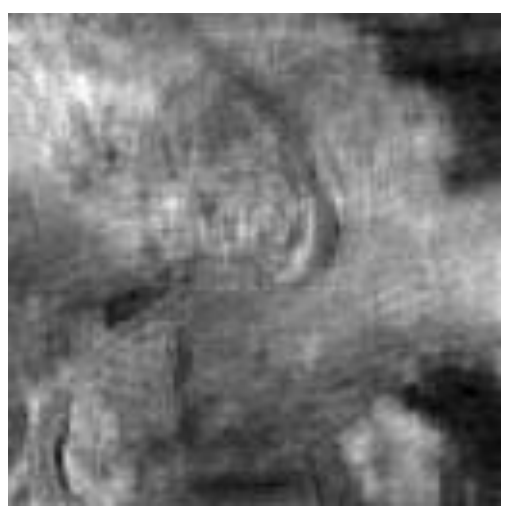

(b)

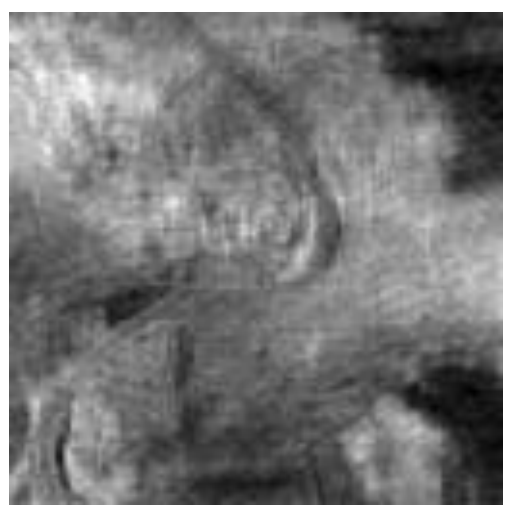

(c)

(b) $\mathrm{MSE}=47.6$

Fig.(11) Reconstructed images of Fig.(8-d) (i.e. $R=2$ and $S N R=30 \mathrm{~dB}$ ) after:
(a) 1 iteration
(b) 10 iterations
(c) 30 iterations 


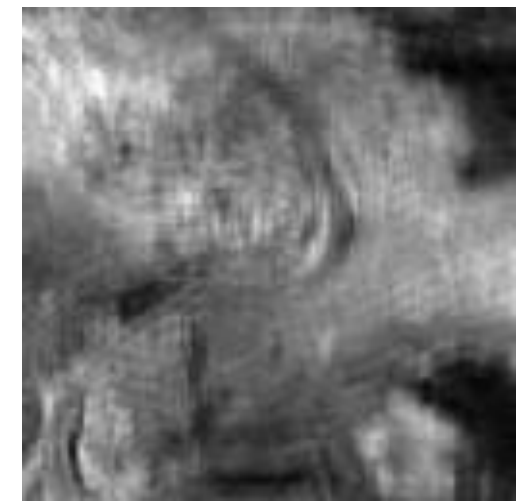

(a)

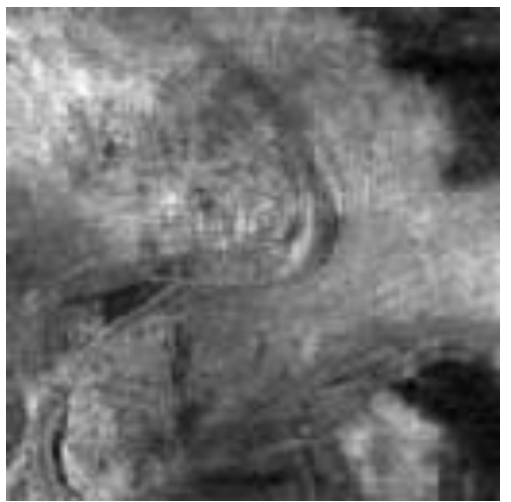

(b)

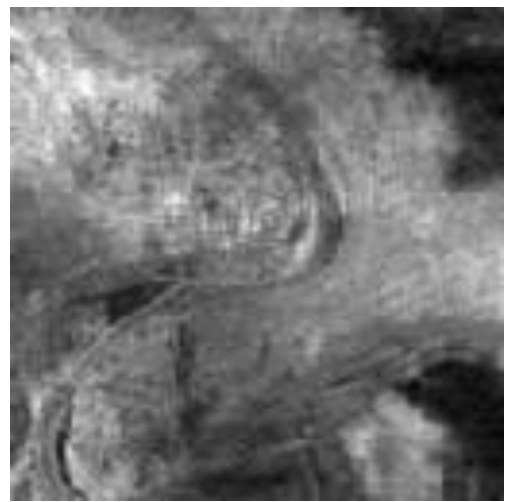

(c)

MSE $=42$

(b) $\mathrm{MSE}=\mathbf{3 0 . 3}$

MSE $=\mathbf{2 7 . 9}$

Fig.(12) Reconstructed images of Fig.(8-e) (i.e. $R=2$ and $S N R=40 \mathrm{~dB}$ ) after: (a) 1 iteration (b) 10 iterations (c) 30 iterations

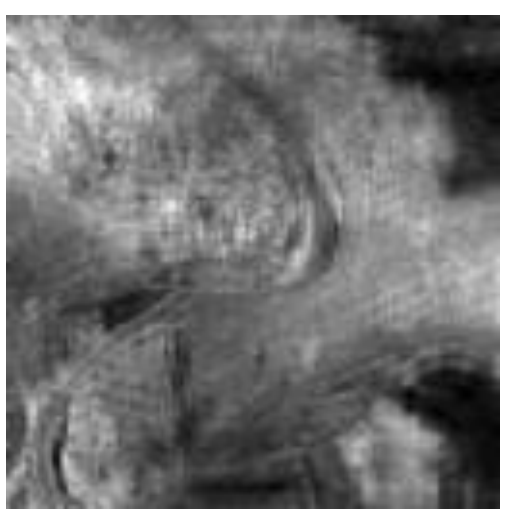

(a)

MSE $=30.9$

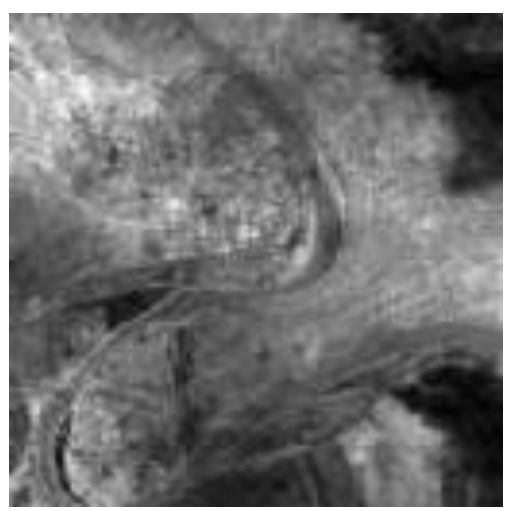

(b)

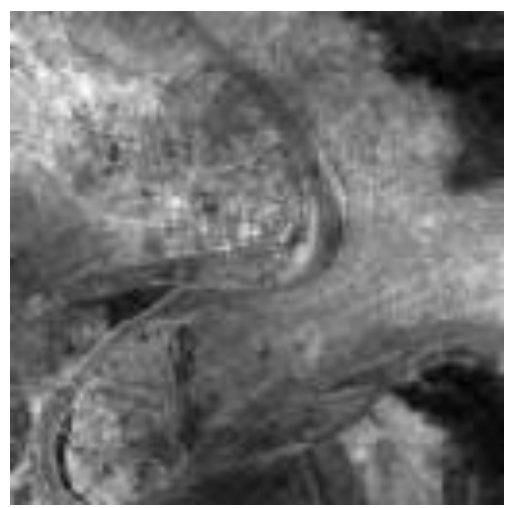

(c)

(b) $\mathrm{MSE}=15.8$

Fig.(13) Reconstructed images of Fig.(8-f) (i.e. $R=2$ and $S N R=50 \mathrm{~dB}$ ) after:
(a) 1 iteration
(b) 10 iterations
(c) 30 iterations 


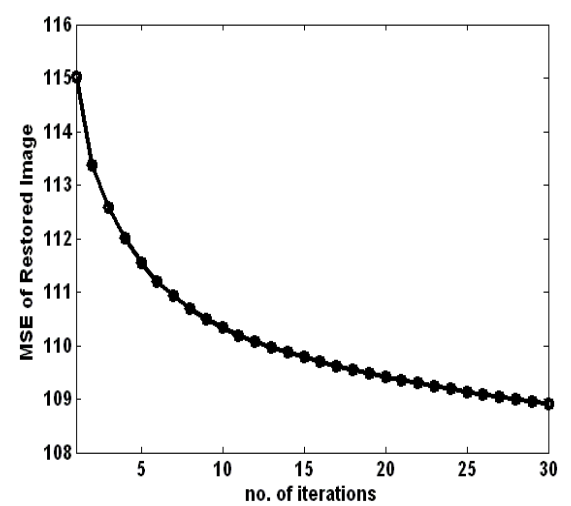

(a)

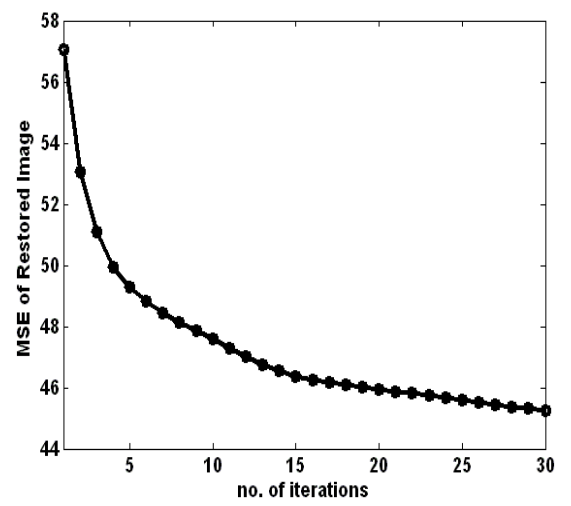

(c)

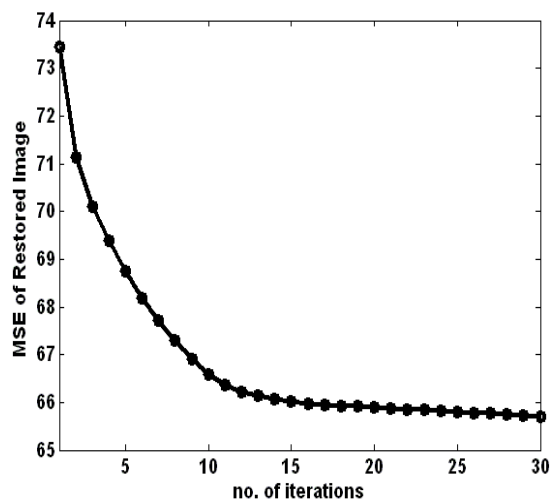

(b)

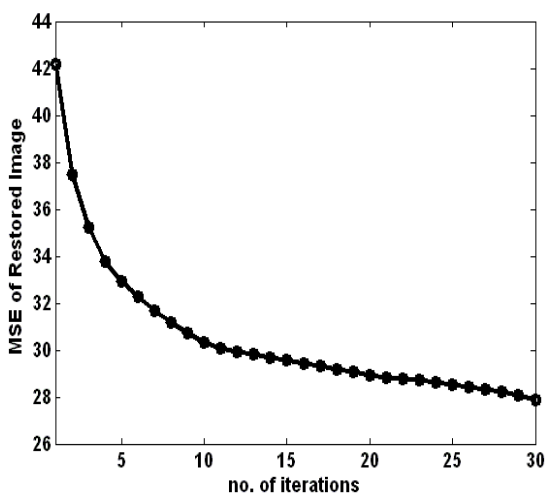

(d)

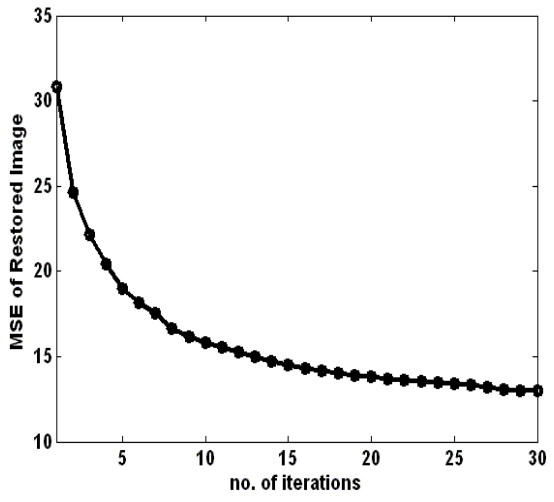

(e)

Fig. (14) Mean Square Errors (MSE) of the Reconstructed images Versus no. of iterations for:
(a) degraded Baghdad image, with circular function of $R=2$ and $S N R=10 \mathrm{~dB}$
(b) degraded Baghdad image, with circular function of $R=2$ and $S N R=20 \mathrm{~dB}$
(c) degraded Baghdad image, with circular function of $R=2$ and $S N R=30 \mathrm{~dB}$
(d) degraded Baghdad image, with circular function of $R=2$ and $S N R=40 \mathrm{~dB}$
(e) degraded Baghdad image, with circular function of $R=2$ and $S N R=50 \mathrm{~dB}$ 